\title{
Cryogenic Cavitation Mitigation in a Liquid Turbine Expander of an Air-Separation Unit through Collaborative Fine-Tuned Optimization of Impeller and Fairing Cone Geometries
}

\author{
Peng Song and Jinju Sun * $\mathbb{B}$ \\ School of Energy and Power Engineering, Xi'an Jiaotong University, Xi'an 710049, China; pengsong@xjtu.edu.cn \\ * Correspondence: jjsun@mail.xjtu.edu.cn; Tel.: +86-029-8266-3777
}

Received: 20 November 2019; Accepted: 16 December 2019; Published: 20 December 2019

check for updates

\begin{abstract}
An air-separation unit (ASU) uses atmospheric air to produce essential pure gaseous and liquid products for many industrial sectors but requires intensive power consumption. In recent years, cryogenic liquid turbine expanders have been used to replace the traditional J-T valves in air-separation units to save energy. In this paper, an effective design optimization method is proposed to suppress swirling flow and mitigate cavitation in liquid turbines. A flexible tuning of the impeller and fairing cone geometries is simultaneously realized, where the optimization variables are identified via a geometric sensitivity study. A novel objective function is deliberately established by allowing both swirling flow and cavitation characteristics, driving the optimizer to search for deswirling and cavitation-resistant geometries. A kriging surrogate model with an adaptive sampling strategy and a cooperative co-evolution algorithm (CCEA) are incorporated to solve the highly nonlinear optimization problem, where the former reduced the costly evaluations but simultaneously maintained the model prediction accuracy and enabled the aim-oriented global searching (the latter decomposes the problem into several readily solved sub-problems that could be solved in parallel at a high-convergence rate). The optimized impeller and fairing cone geometries were quite favorable for suppressing swirling flow and mitigating cavitation. The impeller cavitation was significantly reduced, with the maximal vapor volume fraction reduced from 0.365 to 0.17 at the blade surface; the diffuser tube high-swirl flow was significantly deswirled and the intensive vapor fraction around the centerline largely reduced, with the maximal vapor volume fraction in the diffuser tube reduced from 0.387 to 0.121 . As a result, the isentropic efficiency of the liquid turbine expander was improved from $88.4 \%$ to $91.43 \%$.
\end{abstract}

Keywords: energy saving; air separation unit; cryogenic liquid turbine expander; cavitation; design optimization

\section{Highlights}

- Objective function considers both the swirling flow and cavitation characteristics

- The developed optimization method is proven to be efficient for cavitating flow mitigation

- Collaborative fine-tuned impeller and fairing cone geometries diminish the swirling flow and then suppress cavitation

\section{Introduction}

It is well-known that cryogenic air-separation units (ASUs) are very energy-intensive and require considerable power consumption, and the enhancement of their energy efficiency is in demand. 
In large-scale internal compression ASUs, high-pressure liquefied air (up to 60-75 MPa) needs to be throttled to a low level (around 0.5-0.6 MPa) so as to meet the technical requirements of the downstream distillation column. Conventionally this has been done with a Joule-Thomson valve, but this can cause severe problems. For example, the high-level pressure head is wasted in the cryogenic ASU, and it unexpectedly raises the cryogenic system temperature, which can compromise the ASU's energy efficiency by $2.5 \%-3.5 \%$. In recent years, liquid turbine expanders have been adopted in place of traditional Joule-Thomson valves, as they have a proven capacity for enhancing ASU energy efficiency [1-3]. Liquid turbine expanders reduce the pressure head of the working medium, while simultaneously converting it into output shaft power that can prevent heating from being throttled and further produce a cryogenic refrigeration effect. As a result, the use of a liquid turbine expander can produce significant energy-saving benefits in ASUs, (e.g., in [4-6] the power consumption of the ASU is reduced by $3.1 \%$ ).

Similar to conventional hydraulic turbomachines of pumps and hydraulic turbines, detrimental cavitation also unavoidably occurs in liquid turbine expanders. Cavitation of conventional hydraulic turbomachines has attracted considerable research, but very limited work on cryogenic turbomachines has been reported [7-9].

In both conventional hydraulic turbines and pumps, swirling-flow-induced cavitation is significant and detrimental [10-15]. In hydraulic turbines, swirling flow originates from the rotating impeller, but further propagates downstream into the diffuser tube, leading to considerable mechanical head dissipations [16]. It is also responsible for local cavitation around the impeller exit and draft rube [17-23]. Both experimental and numerical approaches have been used to reveal the relevance between swirling flow and cavitation $[10,24-28]$. The optimized diffuser tube inlet velocity profile has been proven effective for cavitation mitigation [29-33]. The counterpart pump of a hydraulic turbine also suffers from swirling-flow-induced cavitation, but this happens around the impeller inlet and its upstream duct. Previous investigations have demonstrated that modified impeller leading edge geometries can improve pump cavitation performance [34-36]. The use of properly designed inlet inducers has also been effective for pump cavitation mitigation [37,38], and can control cryogenic pump cavitation in liquid rocket propulsion systems [39-41].

In liquid turbine expanders, the swirling vortex downstream the impeller is significant, as it usually operates even at high rotating speeds up to tens of thousands of RPM, which unavoidably results in detrimental swirling-flow-induced cavitation. Notably, cavitation of conventional hydraulic turbines/pumps is mainly associated with local pressure, but cavitation of cryogenic liquid turbine expanders is much more complex, and depends on both the variations of static pressure and static temperature due to the significant thermodynamic effect of cryogenic fluids. Over the years, significant work on cryogenic liquid turbine expanders and the cavitating flow mechanism have been explored by the present team of authors [42-44]. As demonstrated in [44,45], the swirling flow in liquid turbine expanders has significant influence over impeller and diffuser tube cavitation, and both swirling flow and cavitation are sensitive to the geometric variations of impeller blades [44]. In our previous work [46], a fairing cone was introduced to control impeller cavitation. The numerical results show that the cavitating flow is sensitive to the fairing cone geometry variations, indicating that the geometry optimization of fairing cones is essential for cavitation suppression. Should the impeller geometry and fairing cone shape be collaboratively fine-tuned, the cavitation resistance capacity of cryogenic liquid turbine expanders would be largely improved.

The present study focuses on the cavitation mitigation of a cryogenic liquid turbine expander by fine-tuning the geometry optimization of the impeller trailing edge and fairing cone shape. An effective computational fluid dynamic (CFD)-based design optimization method is developed. The objective function is defined considering both swirling flow and cavitation characteristics, which drives the optimizer to search for the right track towards low-cavitation geometries. A kriging model is adopted to reduce the costly and time-consuming CFD evaluations of the objective function, but it is updated with an adaptive sampling strategy to improve prediction accuracy and subsequently help in achieving 
the global optima. The optimization problem is solved by the cooperative co-evolution algorithm (CCEA), which decomposes the complex problem into several simpler sub-problems and solves them in parallel with high-convergence rate. It is proven that the optimized impeller trailing edge geometry and fairing cone shape are favorable for reducing swirling flow and mitigating cavitation.

\section{CFD Models and Validations}

Intensive CFD callings are required for the evaluation of cavitation performance in the optimization process and to establish the objective functions $[13,44,47]$. To have an insight into cavitating flow physics prior to commencing the optimization, two-phase flow analysis is conducted for the originally designed expander geometry, from which the influential geometric factors are identified. The CFD simulations are carried out at the expander stage with ANSYS-CFX.

\subsection{Physical Model and Meshing}

Table 1 presents the main design parameters of the presently investigated cryogenic liquid turbine expander, which uses liquefied air as a working medium.

Table 1. Specifications.

\begin{tabular}{ccc}
\hline Parameters & Units & Value \\
\hline Inlet pressure & $\mathrm{MPa}$ & 7.00 \\
Outlet pressure & $\mathrm{MPa}$ & 0.58 \\
Inlet temperature & $\mathrm{K}$ & 97.15 \\
Mass flow rate & $\mathrm{kg} / \mathrm{s}$ & 26.84 \\
Rotational speed & $\mathrm{rpm}$ & 20,000 \\
\hline
\end{tabular}

The physical model comprises the volute, vanned nozzle ring, shroud impeller and diffuser tube, as shown in Figure 1.

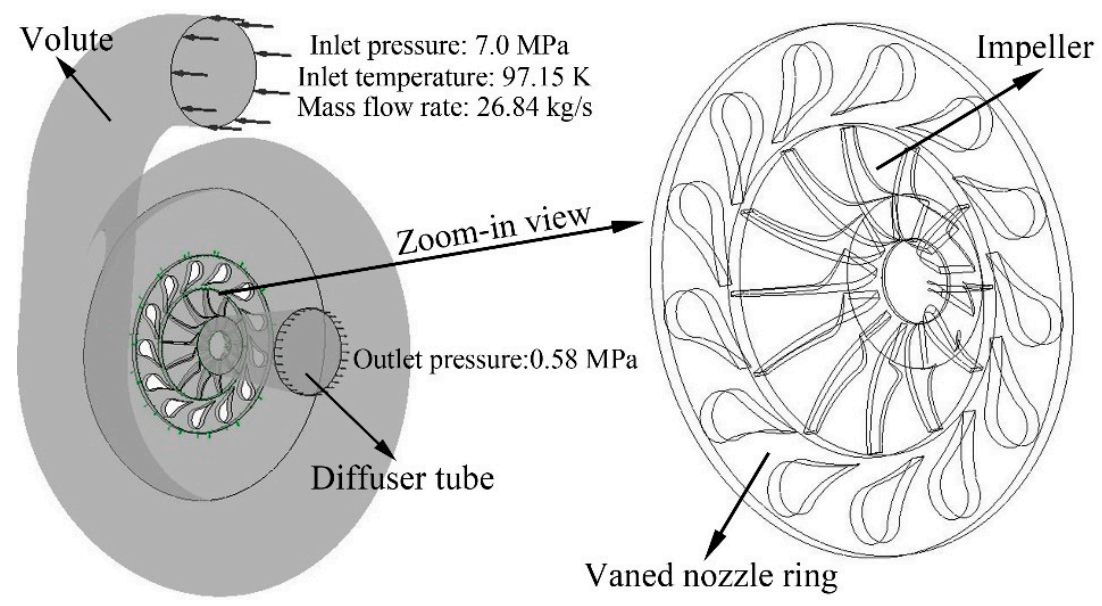

Figure 1. Physical model of the cryogenic liquid turbine expander.

As shown in Figure 2, the hexahedral structured grids are generated for the vanned nozzle and impeller using Turbogrid, the structured grid is generated for the diffuser tube and the unstructured grid for the volute zone using ICEM. To capture the near wall flow behavior, the grids are refined at the blade surfaces, leading and trailing edges and end wall regions. 


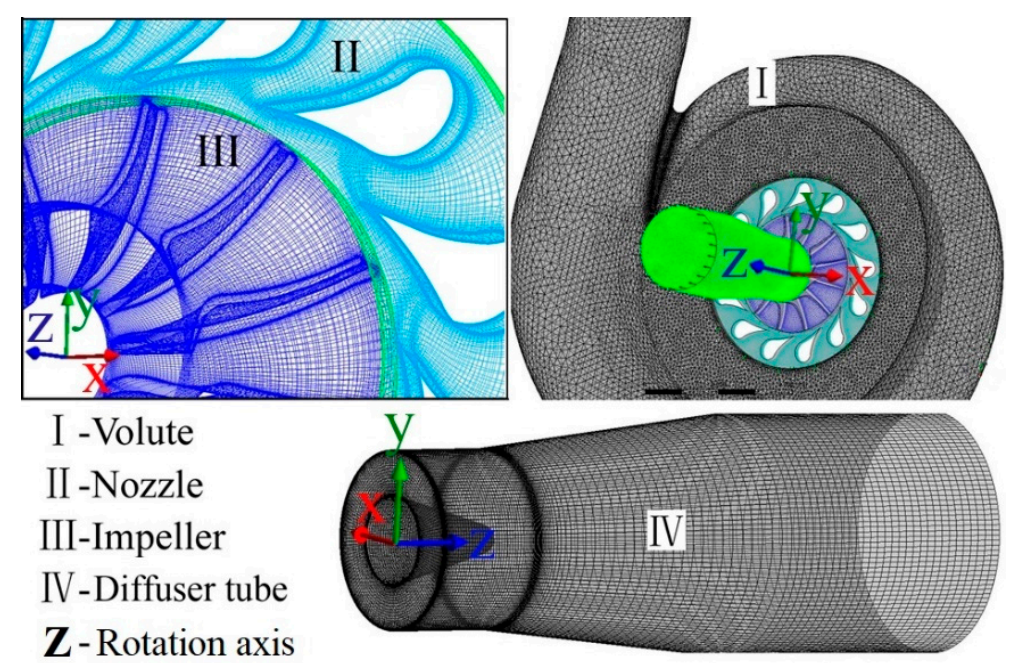

Figure 2. Meshing.

The grid quality is checked by using ICEM software with a minimum face orthogonality angle of $>25^{\circ}$. In the converged (mesh-independent) design optimization solutions, the expansion rates near the hub and shroud boundary layers of both the impeller and exducer are about 1.2, and the mesh expansion rate near the blade surface is around 1.2-1.3. Moreover, once the flow simulation is accomplished, all the $y+$ values have been checked, with the value of $y+$ being less than 10.2 around all the solid boundaries, which satisfies the requirement of the $k-\varepsilon$ model adopted in the present study [48].

\subsection{Physical Properties of Cryogenic Air}

As for cryogenic turbomachines, the cavitation is more complex. This is because cryogenic fluids are more sensitive to temperature variation and the significant latent heat cannot be ignored, whereas both aspects are commonly neglected in conventional hydraulic machines. As mentioned for the cavitation models, the total energy equation is incorporated to allow for temperature variation in the cryogenic flow. Besides, variations are also considered for the thermal physical properties of liquefied air versus both temperature and pressure. In the present study, the thermal properties of liquefied air are calculated by the NIST-REFPROP and represented as the functions of static temperature and pressure to connect with the CFX interface module.

\subsection{Numerical Models}

\subsubsection{Cavitation Model and Validation}

The so-called Rayleigh-Plesset (RP) model [14] is used for cavitating flow simulations. The cavitation model incorporates the homogeneous mixture hypothesis with the Rayleigh-Plesset model, where the former treats the liquid and vapor phases as a continuous mixture, and the latter describes the bubble dynamics $[49,50]$.

The RP equation controls the vapor generation and condensation, as is given by

$$
R_{B} \frac{d^{2} R_{B}}{d t^{2}}+\frac{3}{2}\left(\frac{d R_{B}}{d t}\right)^{2}+\frac{2 \sigma}{\rho_{l} R_{B}}=\frac{p_{v}-p}{\rho_{l}}
$$

where $R_{B}$ is the bubble radius, $p$ is the liquid pressure surrounding the bubble, $p_{v}$ is the pressure in a bubble, $\rho_{l}$ is the liquid density and $\sigma$ is the surface tension coefficient. 
The mass transfer rate is derived from the RP model and expressed as a source term in continuity equations. In CFX, the source term is modeled by employing the Zwart-Gerber-Belamri model as the mass transfer model [51], as presented below.

$$
\dot{m}_{f g}= \begin{cases}F_{v a p} \frac{3 r_{n u c}\left(1-r_{v}\right) \rho_{v}}{R_{B}} \sqrt{\frac{2}{3} \frac{P_{v}-P}{\rho_{l}}} & \text { if } P<P_{v} \\ F_{\text {cond }} \frac{3 r_{v} \rho_{v}}{R_{B}} \sqrt{\frac{2}{3} \frac{P-P_{v}}{\rho_{l}}} & \text { if } P>P_{v}\end{cases}
$$

In the above equations, $r_{n u c}$ is volume fraction at the nucleation site, and $F_{\text {vap }}$ and $F_{\text {cond }}$ are the empirical coefficients for evaporation and condensation processes, respectively. In the present study, these coefficients take the values of $r_{n u c}=5.0 \times 10^{-4}, R_{B}=1.0 \times 10^{-6} \mathrm{~m} F_{\text {vap }}=0.2, F_{\text {cond }}=$ 0.012 , respectively.

Such a model was justified by some previous investigators in the studies of both conventional hydraulic machines [13,52], and cryogenic turbomachines [7-9], with the cavitation models having been verified by the prese $t$ team of authors in our previous publications $[42,44]$. The cryogenic hydrofoil flow was simulated and the predicted surface static pressure and temperature were consistent with the test data [53] (Figure 3). The predicted hydrofoil cavity length over the upper and lower surfaces was $0.0156 \mathrm{~m}$, which is very close to the experimental value of $0.0152 \mathrm{~m}$ by Hord [53].

(a)

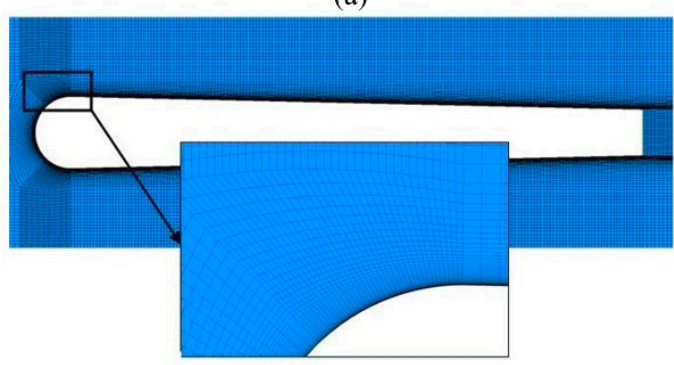

(c)

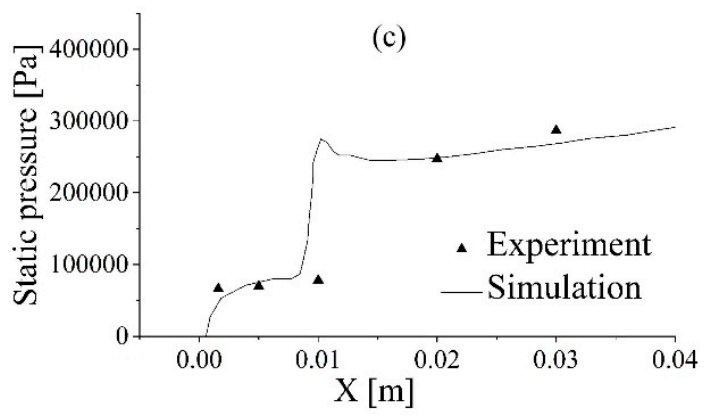

(b)

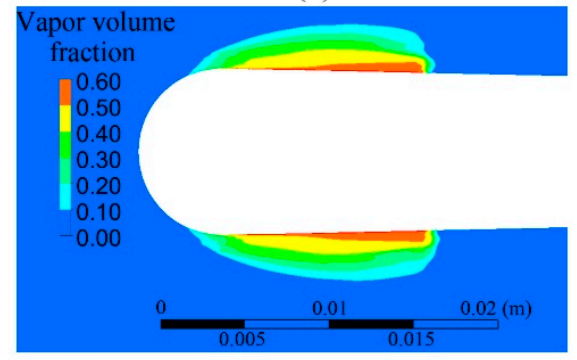

(d)

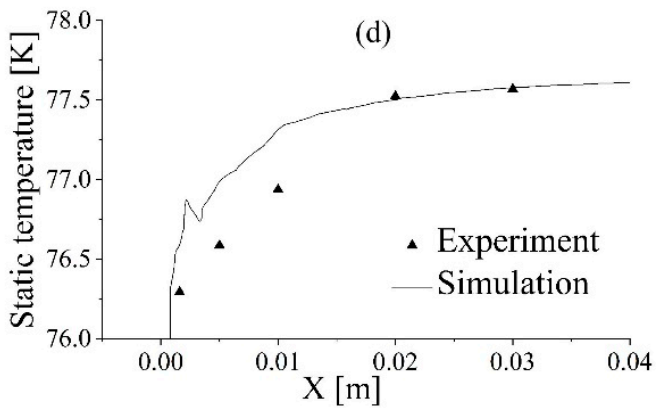

Figure 3. Comparisons of predicted cryogenic hydrofoil cavitating flow with the measured data: (a) computational grid; (b) contour of vapour volume fraction; (c) surface static pressure profile; (d) surface static temperature profile.

\subsubsection{CFD Models}

Flow simulation is conducted with CFX in the staged environment, where the finite-volume method is adopted to discretize the RANS equations and the $k-\varepsilon$ model is adopted with a scalable wall function to close the turbulence terms for a robust convergence. Such a method is justified by the previous tube swirling flow investigations [54,55]. Moreover, a total energy model is adopted, allowing for a thermodynamic effect.

\subsubsection{Boundary Conditions}

As for the inlet and boundaries, total pressure $(7.0 \mathrm{MPa})$ and total temperature $(97.15 \mathrm{~K})$ at the volute inlet are given, while averaged static pressure $(0.58 \mathrm{MPa})$ is specified at the diffuser outlet. 
For the rotational interface of the nozzle-impeller and impeller-diffuser, the frozen rotor approach is specified.

\subsubsection{Convergence Criteria}

Termination of flow simulation is determined according to the information of two successive iterations, and will terminate as the following convergence criteria are satisfied simultaneously:

(1) the difference between inlet and outlet mass flow is less than $1 \%$;

(2) residuals of heat transfer parameters are less than $10^{-4}$;

(3) monitored temperature variations are less than $0.001 \mathrm{~K}$;

(4) variation of shaft power is less than $1 \%$.

The convergence criteria meet the requirement of the engineering applications, offering a compromise between computational accuracy and expense while providing sufficient precision of the predicted temperature to capture the characteristics of the cavitating flow.

\subsubsection{Grid-Independency Study}

To exclude the influence of grid size, the simulation results with different mesh sizes were tested. As shown in Table 2, the grid size of the volute zone was fixed, while the grid size of nozzle, impeller and diffuser tube were gradually increased. The initial grid of $A$ was 2.3 million. Then, three larger grids of $B, C$ and $D$ were obtained through refinement. As shown in Table 2, the sizes of grids increased from $A$ to $D$, where the total sizes of $A, B$ and $C$ are increased linearly.

Table 2. Independence study.

\begin{tabular}{lccllcc}
\hline Cases & Total Number & Volute & Nozzle & Impeller & Diffuser Tube & Runtime [s] \\
\hline A-initial & $2.30 \times 10^{6}$ & & 613,040 & $1,075,340$ & 124,354 & 11,080 \\
B-refined & $3.21 \times 10^{6}$ & 495,382 & 916,007 & $1,617,860$ & 181,653 & 16,960 \\
C-refined & $4.06 \times 10^{6}$ & & $1,208,012$ & $2,130,480$ & 228,708 & 21,520 \\
D-refined & $4.59 \times 10^{6}$ & & $1,389,810$ & $2,444,530$ & 261,678 & 24,588 \\
\hline
\end{tabular}

Numerical simulations were conducted for the four groups of grids, and the predicted expander efficiency, mass flow rate and running time are compared in Table 2 and Figure 4. For the grids less than group $C$, the deviations in overall efficiency were significant; for grid $C$ and $D$, small deviations were produced, but the computation time increased significantly with the grid size.

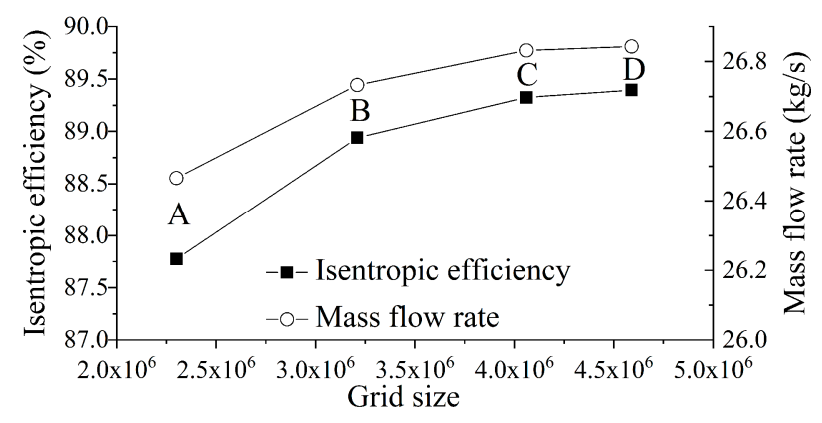

Figure 4. Grid independence.

Thus, grid $C$ with 4.06 million is regarded as reasonable, allowing for both computational accuracy and time, and is used throughout the present study. 


\subsubsection{Numerical Methods Validation}

The above numerical methods were validated by the experimental data in our recent paper [56], in which the flow simulation was conducted with the same parameters as the experimental test.

As shown in Figure 5, the predicted efficiency curve tended to agree well with the experimental data variations, but there was a deviation between the two curves. By consideration the measured error around $\pm 4.0 \%$ reported in [56], the predicted curves reflected the actual trend.

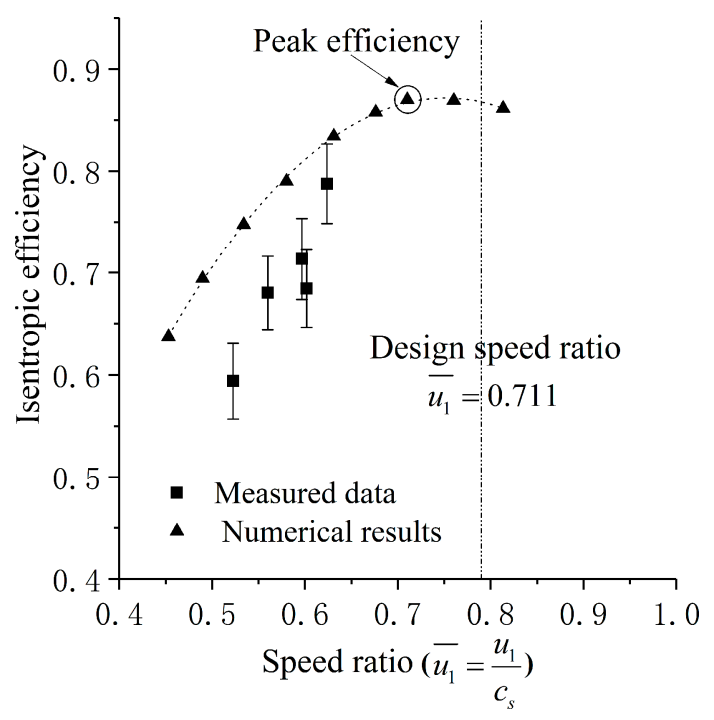

Figure 5. Predicted and measured efficiency curves.

It is noticed that the numerical approach provided more wide-range data than the measured one, and the predicted peak efficiency resided at a speed ratio of 0.71 , which is consistent with the ideal theoretical optimal value suggested in [57] (i.e., $\overline{u_{1}}=1 / \sqrt{2}=0.707$ ).

\subsection{Flow Investigation of the Original Turbine Expander}

With the aforementioned CFD models, numerical simulations were conducted on the original impeller and fairing cone geometries. The obtained results are presented and discussed below.

Figure 6a presents the vapor volume fraction for the impeller and diffuser tube, where the cavitation resided mainly in three regions: region $\mathrm{A}$ at the impeller blade trailing edge, region $\mathrm{B}$ at the diffuser tube inlet and region $C$ around the diffuser tube centerline. The impeller trailing edge cavitation resulted from the mismatched flow behavior, where the blade trailing geometry did not suit the local exit flow, causing the flow to separate from the blade surface, as depicted by the streamline contour on the left of Figure $6 \mathrm{~b}$. Such flow separation caused local mechanical head dissipation, as indicated by the entropy contour. Subsequently, local pressure drops and temperature rises occurred, as shown in middle and right of Figure 6b, respectively. The combined effect of low static pressure and high static temperature was responsible for the local cavitation. 
(a)
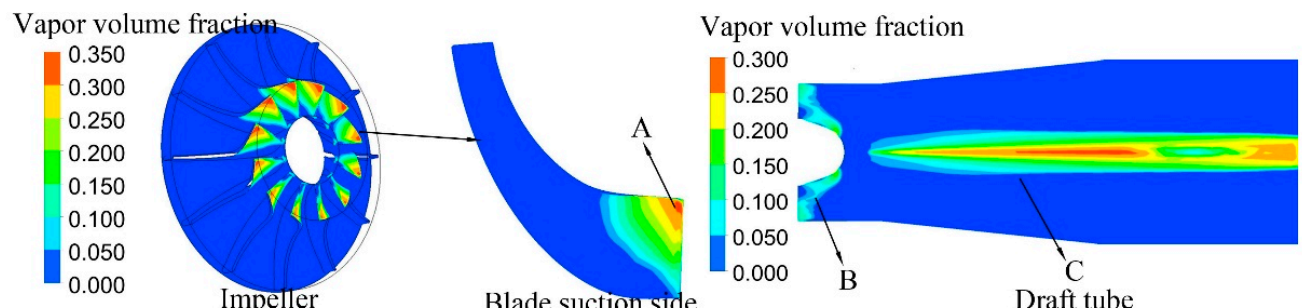

(b)
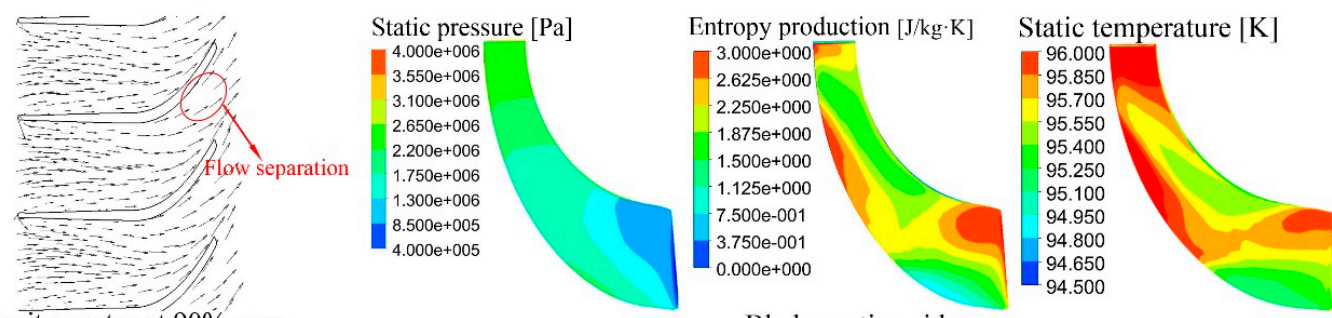

Velocity vector at $90 \%$ span

Blade suction side

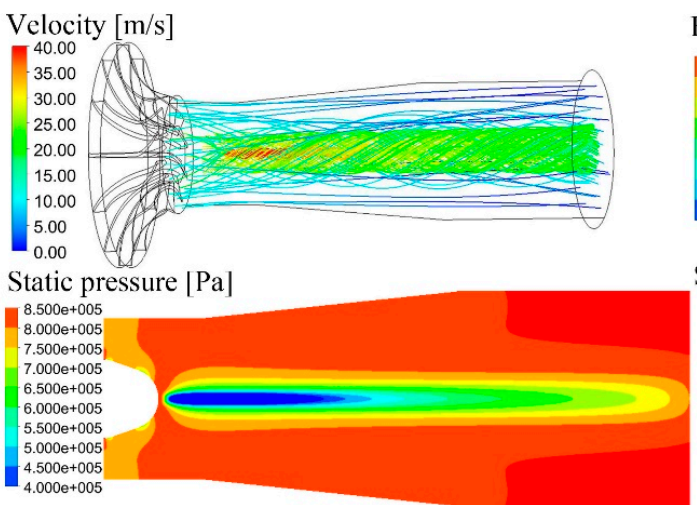

Entropy production $[\mathrm{J} / \mathrm{kg} \cdot \mathrm{K}]$

(c)

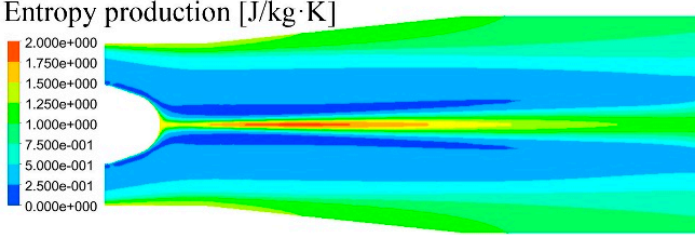

Static temperature $[\mathrm{K}]$

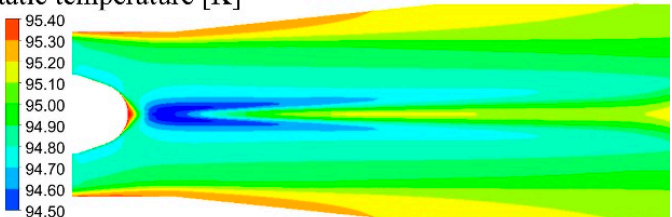

Figure 6. Flow behavior in the original designed expander impeller and diffuser tube: (a) impeller and diffuser tube vapor volume fraction; (b) impeller flow; (c) diffuser tube flow.

Cavitation in the diffuser tube occurred in two regions and of different scales seen in Figure 6a (right side): the smaller formed downstream from the impeller trailing edge, while the larger formed around the centerline. The former resulted from the propagation of impeller cavitation while the latter resulted from the diffuser tube swirling flow. As shown by the streamline/velocity contour in Figure $6 c$, the impeller exiting flow further developed into a rope-like swirling flow in the vaneless diffuser tube due to the significant circumferential component of the impeller exiting-velocity-produced high-speed rotation. Such vortex swirling flow led to considerable flow losses around the center core region, as depicted by the entropy production contour and subsequently the local-pressure drops and temperature-rises shown above. The combination of low static pressure and high static temperature was responsible for the swirling-flow-associated cavitation around the diffuser tube center core region.

Obviously, to suppress the expander cavitation, reducing the impeller trailing edge flow separation and deswirling the diffuser tube vortex flow are essential. This can be achieved by a collaborative fine-tuned geometric optimization of the impeller exit and diffuser tube fairing cone shapes, as introduced in the following sections.

\section{Geometric Design Optimization Method}

An effective optimal design method was developed for cavitating flow suppression, which involves a suitable objective function, identifying the optimization variables and solving the highly nonlinear complex design optimization problem globally and efficiently. 


\subsection{Optimization Variables}

In the optimization, a flexible tuning of the impeller geometry and fairing cone shape was simultaneously realized, with the former tuned at both levels of the 2D meridian profiles and 3D blade geometry, and the latter tuned in 2D dimensions. The optimization design variables were identified on a basis of geometric design analysis and sensitivity study.

As shown in Figure 7, the impeller meridional was defined by two elliptic parabolic curves of 2-3 and 1-4, and their variations were realized by adjusting $R_{1}, R_{2}, R_{4}, b, L$, and $\alpha_{1}$. The impeller comprises a radial-straight work wheel and the twist exducer, where the latter is of a cylinder-parabola curved surface and parameterized by three blade angles of $\theta_{0 M}, \beta_{B 0 M^{\prime}}^{0}, \beta_{B 02}^{0}$. The fairing cone is used to make a gradual transition of the flow path from the impeller blade passage to the vaneless diffuser tube. The fairing cone head is a sphere of diameter $D_{0}$, which is tangent to the line $0-1$, while $\beta$ denotes the taper angle.
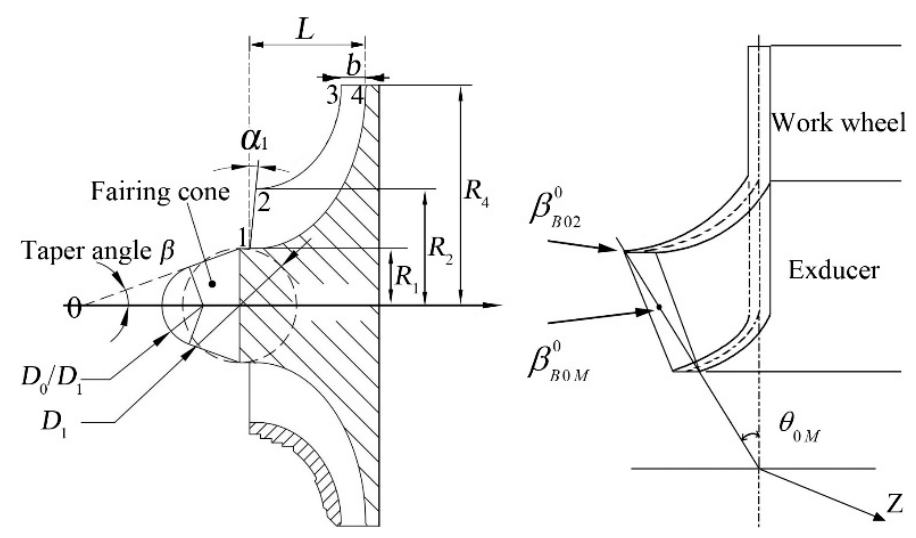

Figure 7. Geometric parameterization.

Sensitivity studies were conducted for the impeller blade and fairing cone geometric parameters to obtain the most flow-sensitive geometric parameters. These are then used as the design variables for design optimization.

In total, eight geometric parameters were identified as design variables, including $R_{1}, R_{2}, \alpha_{1}$, $\theta_{0 M}, \beta_{B 0 M^{\prime}}^{0} \beta_{B 02}^{0}, \beta$ and $D_{0} / D_{1}$. The first three variables represent the meridian profile variations, where $R_{1}$ denotes the impeller outlet inner radius, $R_{2}$ the impeller outlet outer radius and $\alpha_{1}$ the biased angle of the inducer external end-face. The second three variables describe the impeller blade twist, where $\theta_{0 M}$ denotes the maximal circumferential twist angle, $\beta_{B 0 M}^{0}$ the blade angles at mean radius and $\beta_{B 02}^{0}$ the blade angles at the tips. The final two variables describe the fairing cone shape variation, where $\beta$ denotes the fairing cone taper angle, and $D_{0} / D_{1}$ the normalized fairing cone head diameter. The above eight geometric variables were collaboratively optimized by the developed optimization method, which is presented below.

\subsection{Establishment of Objective Function}

For turbomachine design optimization, a well-suited objective function is essential. Commonly, the overall performance of the efficiency and pressure ratio is set as the optimization goal. However, this can compromise the optimization benefits, as the flow characteristics are excluded from the optimization target, whereas they are the ultimate basis for achieving high-level overall performance. If the design goal is to control the flow (for instance, the present study is aimed at cavitation suppression), then setting the overall performance as the objective function obviously will compromise the optimization benefits.

In this study, a novel objective function was established to consider both swirling flow and cavitation characteristics, driving the optimizer to search for the right track towards low-cavitation geometries. It takes two steps to establish such an objective function: characterizing the swirling and cavitation flows, and establishing the objective function. 


\subsubsection{Flow Characterization}

With each of various combinations of impeller and firing cone geometries, the liquid turbine expander flow and overall performance were predicted, respectively, and the relations of the flow and the overall performance parameters are herein explored. On such a basis, the characteristic parameters are determined, which are $\eta, V f_{\text {ave }}, \zeta$ and $V c_{\text {ave }} . \eta$ represents the overall isentropic efficiency of a turbine expander, and is used to prevent an excessive sacrifice in overall performance with various geometric combinations; $V f_{\text {ave }}$ is the averaged vapor volume fraction at the impeller blade suction surface, depicting the impeller cavitation intensity and scale; $V c_{\text {ave }}$ is the averaged circumferential velocity component of the diffuser tube flow depicting the flow swirling intensity in the diffuser tube; $\zeta$ is the loss coefficient of the diffuser tube flow depicting the loss level, which is obtained from the total pressure over the cross sections of the diffuser tube inlet and outlet.

$$
\zeta=\frac{\frac{1}{A_{\text {in }}} \int_{\text {in }} P_{t} d A-\frac{1}{A_{\text {out }}} \int_{\text {out }} P_{t} d A}{\frac{1}{2} \rho\left(\frac{Q}{A_{\text {in }}}\right)^{2}}
$$

where $P$ denotes the static pressure; $P_{t}$ is the total pressure and $P_{t}=P+0.5 \rho\left(u^{2}+v^{2}+w^{2}\right)$, being normalized using the energy flux estimator $\frac{1}{2} \rho\left(\frac{Q}{A_{\text {in }}}\right)^{2}$.

The flow characteristic parameters varied accordingly with the geometric parameters of impeller blade and fairing cone shapes, and they also depicted the expander flow behavior, which is illustrated through the following example cases.

Table 3 presents the flow characteristic parameters obtained respectively from four different combinations of impeller and fairing cone geometries. As the geometric parameters varied within their ranges, each flow characteristic parameter (except the overall performance indicator of $\eta$ ) varied significantly within its own range, demonstrating the sensitive relationship between geometric and characteristic parameters. The variations of the characteristic parameters represented the corresponding variations in the flow behaviors. As shown in Figure 8a, the maximal and minimal vapor volume fraction of the four cases occurred in cases 1 and 2, respectively. According to the scale and intensity of impeller cavitation, the cases could be sorted from strong to weak in the following order: case 1, 4, 3 and 2. The same orders are shown by the predicted characteristic parameter $V f_{\text {ave }}$ in Table 3.

Table 3. Characteristic and overall performance parameters versus geometric parameters.

\begin{tabular}{|c|c|c|c|c|c|c|c|c|c|}
\hline \multicolumn{2}{|c|}{ Parameters } & Symbol & Units & Range & Original & Case 1 & Case 2 & Case 3 & Case 4 \\
\hline \multirow{4}{*}{\multicolumn{2}{|c|}{$\begin{array}{l}\text { Flow characteristic parameters } \\
\text { and overall performance } \\
\text { indicator }\end{array}$}} & $\eta$ & - & $0.8761-0.9039$ & 0.8839 & 0.8984 & 0.8891 & 0.8826 & 0.8971 \\
\hline & & $V f_{\text {ave }}$ & - & $0.0193-0.0251$ & 0.0238 & 0.0243 & 0.0198 & 0.0225 & 0.0231 \\
\hline & & $\zeta$ & - & $0.0276-0.0571$ & 0.368 & 0.427 & 0.265 & 0.371 & 0.322 \\
\hline & & $V c_{\text {ave }}$ & $\mathrm{m} / \mathrm{s}$ & $1.632-2.773$ & 2.127 & 2.44 & 0.844 & 0.914 & 1.274 \\
\hline \multirow{8}{*}{$\begin{array}{l}\text { Geometric } \\
\text { parameters }\end{array}$} & \multirow{3}{*}{$\begin{array}{l}\text { Meridian } \\
\text { profile }\end{array}$} & $R_{1}$ & $\mathrm{~mm}$ & $9-12$ & 10.4 & 12.0 & 10.5 & 10.0 & 9.5 \\
\hline & & $R_{2}$ & $\mathrm{~mm}$ & $20-23$ & 21.3 & 23.0 & 20.0 & 21.0 & 20.5 \\
\hline & & $\alpha_{1}$ & degree & $-5.0-5.0$ & 0.0 & 4.0 & 2.0 & -1.0 & -4.5 \\
\hline & \multirow{3}{*}{ Blade shape } & $\theta_{0 M}$ & degree & $14.0-28.0$ & 20.0 & 15.0 & 21.0 & 27.0 & 25.0 \\
\hline & & $\beta_{B 02}^{0}$ & degree & $20-30$ & 25.0 & 28.0 & 20.0 & 20.0 & 22.0 \\
\hline & & $\beta_{B 0 M}^{002}$ & degree & $30-40$ & 33.0 & 35.0 & 30.0 & 30.0 & 38.0 \\
\hline & \multirow{2}{*}{$\begin{array}{c}\text { Fairing } \\
\text { cone shape }\end{array}$} & $\beta$ & degree & $15-25$ & 18.0 & 15.3 & 16.5 & 16.8 & 18.5 \\
\hline & & $D_{0} / D_{1}$ & - & $0.2-0.96$ & 0.95 & 0.29 & 0.48 & 0.30 & 0.33 \\
\hline
\end{tabular}


(a)

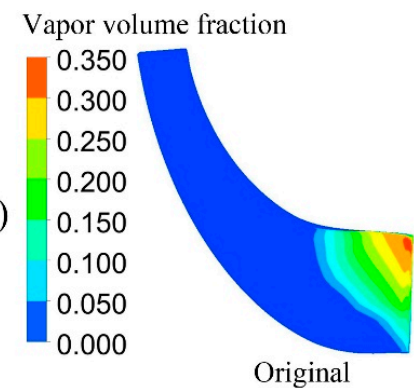

(b)

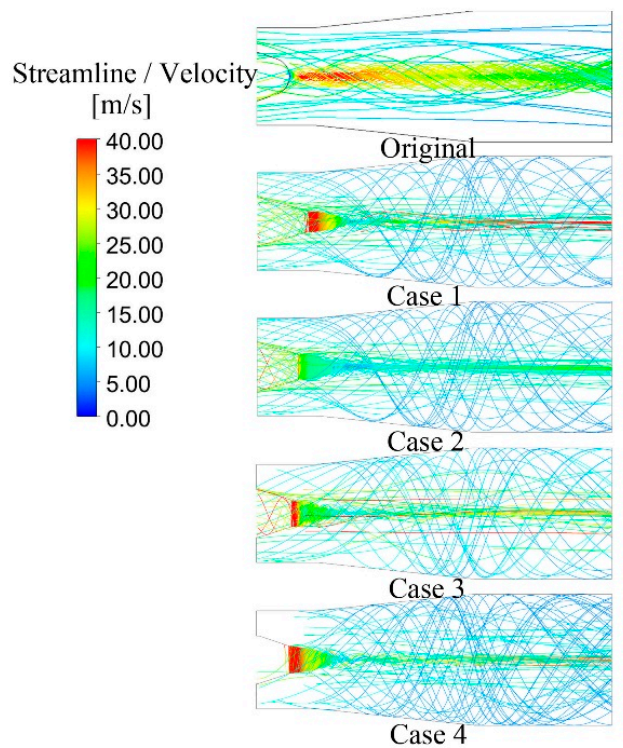

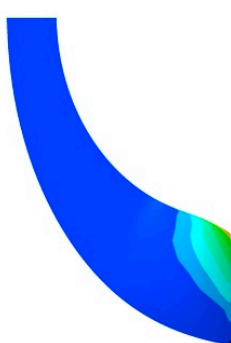

Case 2

Vapor volume fraction

0.250

$-0.200$

0.150

0.100

0.050

0.000
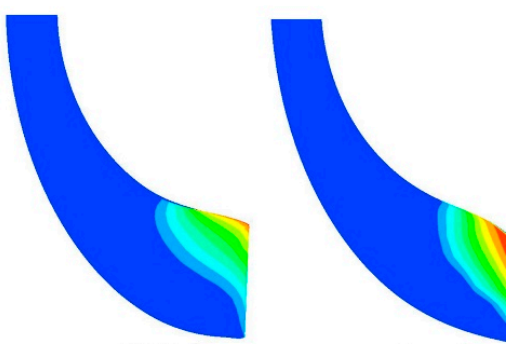

Case 3

Case 4

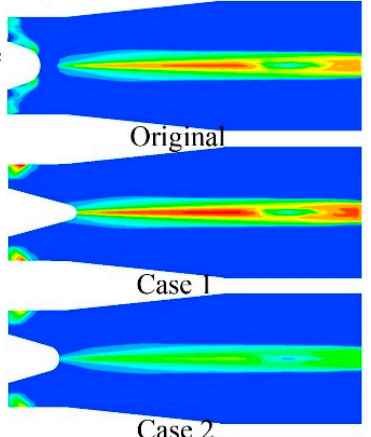

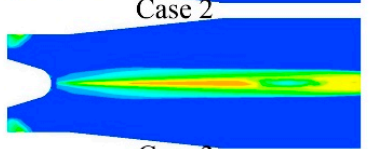

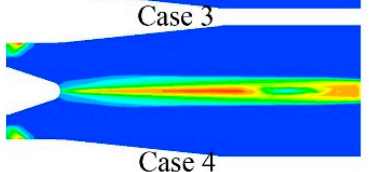

Case 4

Figure 8. Predicted flow patterns and vapor volume fractions for different geometric combinations: (a) cavitation at impeller blade suction side; (b) diffuser tube flow.

The diffuser tube cavitation intensity is shown on the left side of Figure 8 from top to bottom, with cases 1, 4, 3 and 2 representing the highest to lowest intensity, respectively, while the predicted diffuser tube averaged circumferential velocity components of $V c_{\text {ave }}$ in the same order in Table 3, indicating that the diffuser tube cavitating flow mainly resulted from its swirling flow. This was further confirmed by the predicted diffuser tube streamline/velocity contours (right side of Figure 8b), where cases 1, 4, 3 and 2 had the highest to lowest diffuser rope-like flow intensity, respectively, which is identical to the diffuser tube cavitation intensity.

Moreover, the variations of the geometric parameters in cases 1-4 brought about slight changes in the overall efficiency of $\eta(\leq 0.0065)$, as shown in Table 3 , which indicates a less-sensitive relationship between geometric parameters and the overall performance. Clearly, if the overall performance of the turbine expander were used merely to establish the objective function, the design optimization would become ineffective.

\subsubsection{Objective Function Establishment}

As discussed above, flow characteristics in the turbine expander were well-depicted by the four characteristic parameters of $\eta, V f_{\text {ave }}, \zeta$ and $V c_{\text {ave }}$. To achieve the swirling flow control and cavitation suppression goal, the objective function can be defined through a combination of normalized characteristic parameters, given by

$$
o b j(x)=C_{1}(1-\eta)^{\prime}+C_{2} V f_{a v e}{ }^{\prime}+C_{3} \zeta^{\prime}+C_{4} V c_{a v e}
$$

where $x$ is the design variable vector involving the impeller and fairing cone geometry parameters $R_{1}$, $R_{2}, \alpha_{1}, \theta_{0 M}, \beta_{B 0 M^{\prime}}^{0} \beta_{B 02}^{0}, \beta$ and $D_{0} / D_{1}$. 
$C_{1}, C_{2}, C_{3}, C_{4}$ are the empirical coefficients that represent the weights among the four characteristic parameters in Equation (4). In the present study, the four characteristic parameters were normalized in the following equations, respectively, and thus the weight coefficients are assigned to the identical values (i.e., $C_{1}=C_{2}=C_{3}=C_{4}=0.25$ ).

$$
\begin{aligned}
& (1-\eta)^{\prime}=\frac{\max (\eta)-\eta}{\max (\eta)-\min (\eta)} ; \quad V f_{\text {ave }}{ }^{\prime}=\frac{V f_{\text {ave }}-\min \left(V f_{\text {ave }}\right)}{\max \left(V f_{\text {ave }}\right)-\min \left(V f_{\text {ave }}\right)} \\
& \zeta^{\prime}=\frac{\zeta-\min (\zeta)}{\max (\zeta)-\min (\zeta)} ; \quad V c_{\text {ave }}{ }^{\prime}=\frac{V c_{\text {ave }}-\min \left(V c_{\text {ave }}\right)}{\max \left(V c_{\text {ave }}\right)-\min \left(V c_{\text {ave }}\right)}
\end{aligned}
$$

In the normalization Equation (5), the minimum and maximum values of each parameter are used (Table 3), and they are pre-evaluated through CFD simulations before the optimization is commenced.

\subsection{Optimization Framework}

CFD-based turbomachine design optimization is known as a highly nonlinear problem. However, for the cryogenic liquid turbine design optimization, the complexity is increased when cryogenic cavitation and thermodynamic effects are involved, which makes calculating the CFD very costly and time-consuming. During the optimization process, intensive callings of such computationally expensive CFD simulations are required, during which the two-phase swirling and cavitation flow will be predicted, and the objective function will be calculated. Such an optimization can become unaffordable in regards to both time and capital aspects.

In the present developed optimization method, a kriging model was adopted to reduce the cost of CFD evaluations, and it was updated with an adaptive sampling strategy to continually improve its prediction accuracy, so as to achieve a global optimum. The optimization problem was solved using the cooperative co-evolution algorithm (CCEA), which decomposes the original problem into several readily solved sub-problems, then solves them with a high-convergence speed.

The optimization algorithms proposed by the present authors has been successfully applied in axial flow compressors [58] and in cryogenic liquid turbine expander design optimization [44]. The incorporation of such optimization algorithms into the present turbine expander design optimization is briefed below, and a detailed description of the optimization algorithms can be found in our previous publications $[44,58]$.

Figure 9 presents the optimization framework for cryogenic cavitation mitigation in an ASU liquid turbine expander, which is driven by the newly established cavitation-attenuation objective function that allows for the collaborative fine-tuning of the impeller and fairing cone geometric shapes. The optimization methodmainly involves four modules: 'DOE (Design of Experiment)', 'Automatic CFD Evaluation', 'Adaptive Sampling', and 'Database'. Its implementation is briefed below.

(1) At first, 'DOE (Design of Experiment)' is performed, in which quite a number of impeller and fairing cone geometric shape candidates are obtained with a space-filling scheme. CFD simulations are respectively conducted for each of them. The objective functions are then predicted by means of Equation (4) and used to construct the initial 'Database'.

(2) Using the evaluated DOE samples, a coarse kriging-based surrogate model is established.

(3) The optimization loop starts with 'Adaptive Sampling'. The CCEA optimizer and adaptive kriging model are incorporated, with the CCEA solving the auxiliary optimization problem to select new impeller and fairing cone design candidates with a good balance between the optimal searching and surrogate model updates. As the optimization loop proceeds, the potential candidates are successively obtained by the adaptive sampling strategy through the above procedure.

(4) During the optimization, if it is required, 'Automatic Evaluation' will be evoked by a batch code, where the geometric meshing and cryogenic cavitation flow simulation can be set up automatically. Once the simulation is done, the objective function is calculated using Equation (4) and used to update the 'Database'.

(5) The optimization terminates as the prescribed maximal CFD callings are reached. 


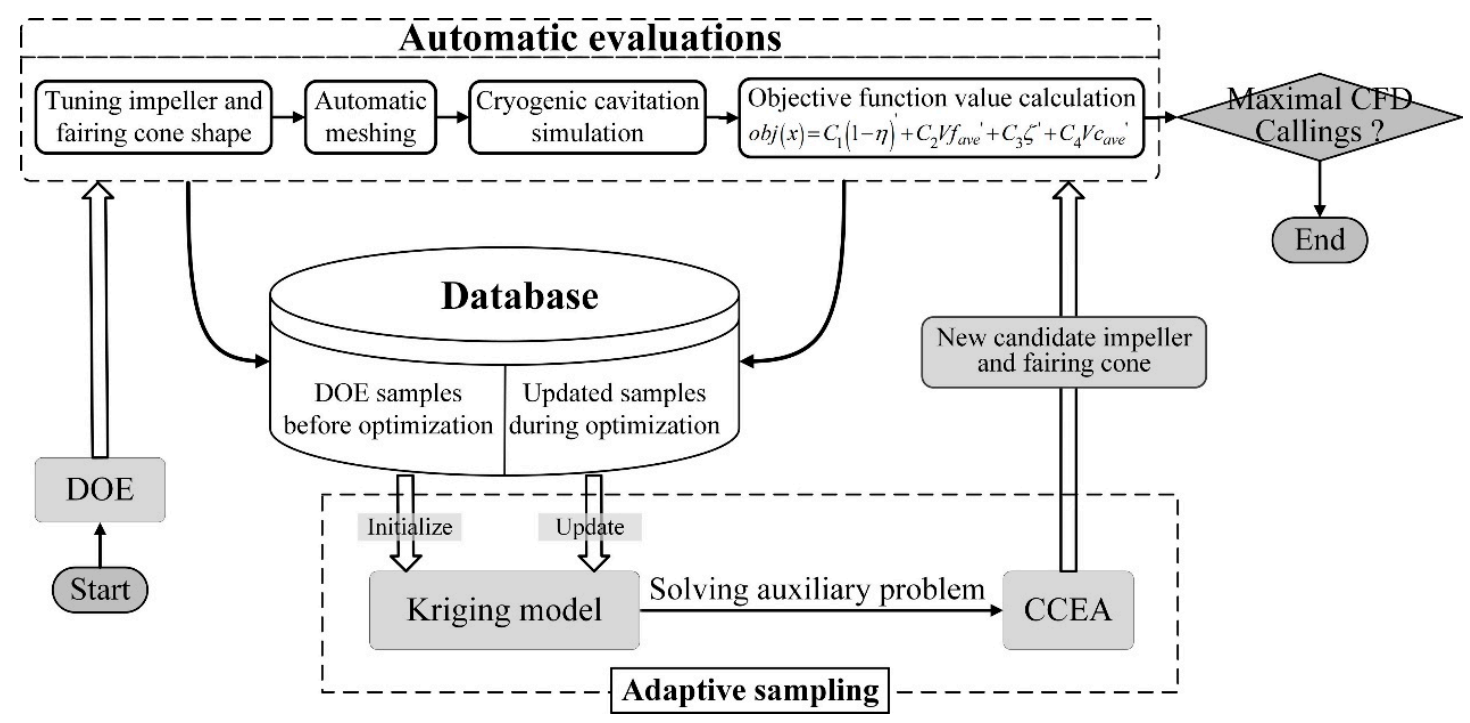

Figure 9. Flowchart of optimization framework.

In the optimization, the adaptive feature of the surrogate model and the parallel capacity of the optimization algorithm both play a significant role. The former helps to reduce the costly CFD evaluations, while simultaneously enhancing the surrogate model prediction accuracy, and this permits the aim-oriented global optimization searching; the latter (i.e., the parallel capacity of the CCEA) accelerates the optimal solution-searching rate. With both, the mitigation of cryogenic cavitation in a liquid turbine expander is made possible through collaborative fine-tuned geometries of the impeller and fairing cone shapes.

\section{Optimization Results}

The optimization was conducted on a single Dell 7500 workstation (with 2 Xeon $2.93 \mathrm{GHz}$ processors and $24 \mathrm{~GB}$ ram), and took some $300 \mathrm{~h}$ to converge. In total, 60 callings of CFD simulations were performed, with 24 callings performed for evaluating the DOE samples being used in the coarse surrogate model construction, and the the other 36 callings are performed for evaluating the updated samples throughout the optimization process.

In the optimization, the impeller blade geometry and faring cone shape were both varied and optimized. Table 4 presents the optimized geometric parameters alongside the original ones. The first three represent the impeller meridian, the middle three describe the 3D blade angles and the last two describe the fairing cone shape.

Table 4. Original and optimized impeller and fairing cone geometric parameters.

\begin{tabular}{ccccc}
\hline Geometry & Parameters & Units & Original & Optimized \\
\hline \multirow{3}{*}{ Meridian profile } & $R_{1}$ & $\mathrm{~mm}$ & 10.4 & 11.2 \\
& $R_{2}$ & $\mathrm{~mm}$ & 21.3 & 21.8 \\
& $\alpha_{1}$ & degree & 0 & 2.6 \\
\hline \multirow{3}{*}{ 3D blade angles } & $\theta_{0 M}$ & degree & 20 & 23.2 \\
& $\beta_{B 02}^{0}$ & degree & 25 & 26.2 \\
& $\beta_{B 0 M}^{0}$ & degree & 33 & 35.1 \\
\hline \multirow{2}{*}{ Fairing cone shape } & $\beta$ & degree & 18.0 & 22.3 \\
& $D_{0} / D_{1}$ & - & 0.950 & 0.454 \\
\hline
\end{tabular}

The change of first three variables indicates significant variations of the hub and shroud profiles. Table 4 shows that the optimized impeller outlet width $(21.8-11.2=10.6 \mathrm{~mm})$ was smaller than the original one $(21.3-10.4=10.9 \mathrm{~mm})$, indicating that a narrower flow passage with a larger averaged 
outlet radius was obtained through optimization. Such variations of flow passage are favorable for improving the blade trailing edge flows, as will be discussed below. The optimized 3D blade angle parameters are also compared with original parameters in Table 4, indicating significant tuning of the 3D blade shape. Moreover, as shown in the table, the fairing cone geometry shape also varied significantly, indicating that its geometric shape was highly associated with the swirling and cavitating flows.

Such geometric variations are driven by the deliberately established objective function and based on flow characteristics. They are proven to be efficient for mitigating the swirling vortex and cavitation, as introduced below.

Figure 10 presents the optimization path of the overall expander performance and characteristic parameters. As shown in the figure, with the original design (of zero CFD evaluation), the impeller's overall efficiency $\eta$ was $88.39 \%$, while the flow characteristic parameters of the impeller suction-side averaged vapor volume fraction $V f_{\text {ave }}$, the diffuser tube loss coefficient $\zeta$ and the circumferential velocity component $V c_{\text {ave }}$ were $0.0238,0.0368$ and 2.127 , respectively. The first 24 CFD calculations were used for DOE sample evaluations, for which the expander overall efficiency and the values of flow characteristic parameters $\left(V f_{\text {ave }}, \zeta\right.$ and $\left.V f_{\text {ave }}\right)$ were both randomly distributed, exhibiting the exact space-filling feature of the DOE samples.

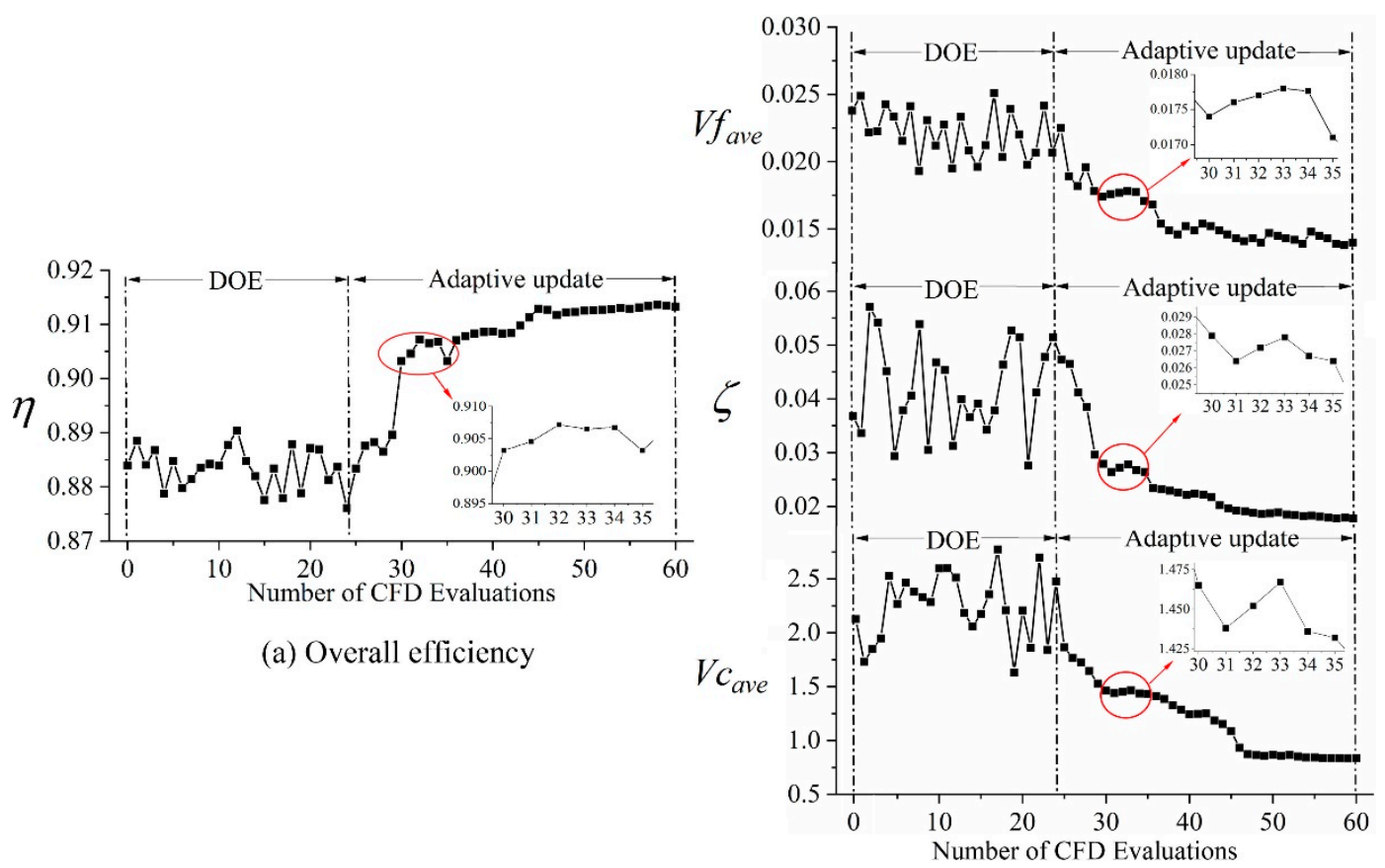

(b) Flow charactersistic parameters

Figure 10. Evolution of the overall efficiency and flow characteristic parameters during optimization: (a) overall efficiency; (b) flow characteristic parameters.

As the optimization proceeded and the CFD evaluations increased, the impeller's overall efficiency rose and the vapor fraction decreased as expected, from 50 to 60 evaluation. All four curves of the optimization path tended to be flat, indicating convergence of the optimization.

The optimization paths in Figure 10 indicate the distinct features of our developed optimization method. As shown in Figure 10b, with the original design (of zero CFD evaluation), the diffuser tube circumferential velocity component $V c_{\text {ave }}$ was of a high level, indicating that the diffuser tube swirling flow was significant. The large loss coefficient $\zeta$ on the curve indicates that there was considerable mechanical head loss. As the CFD evaluations increased during the optimization, the diffuser tube circumferential velocity component decreased; thus, the loss coefficient decreased. In the zoom-in region from CFD evaluations 30 to 32, the overall efficiency and impeller vapor volume fraction all increased, while the diffuser tube loss coefficient and circumferential velocity component decreased 
and then increased in the reverse direction from the optimal. This demonstrates the precise exploitation capacity of the adaptive optimizer when optimal searching is performed in the most infeasible region to prevent being trapped around the local optima. The combination of the descending exploration and ascending exploitation capacity allowed the optimization to converge efficiently at a global solution. From evaluation 50 to 60 , all the curves tended to be flat, indicating the eventual convergence of the optimization.

The optimization path of the quantitative characteristic parameters was also supported by the evolution of cavitation behavior during the optimization process. Figure 11 presents impeller cavitation snaps at their original state and during four different optimization loops.

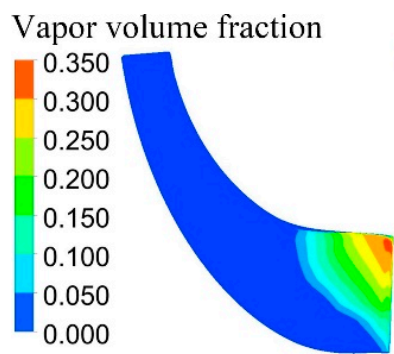

Original

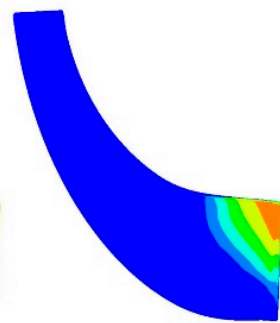

24 evaluations

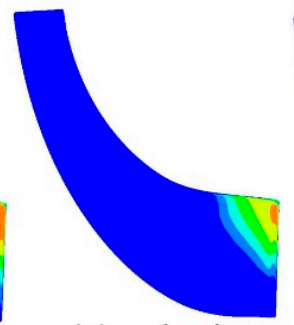

36 evaluations

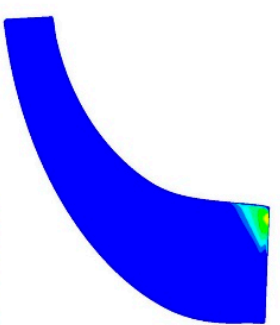

48 evaluations

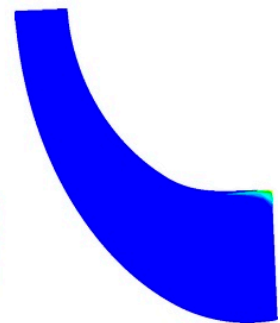

60 evaluations

Figure 11. Evolution of cavitation during optimization process.

As shown above, significant cavitation resided at the trailing edge of original impeller blade. The first 24 evaluations were used for DOE sample evaluations, and the presented results represent the impeller with smallest value $V f_{\text {ave }}$ of the 24 DOE samples. As the optimization proceeded, the scale and intensity of cavitation reduced gradually. For instance, the results from 36 evaluations showed a significantly reduced cavitation, and the cavitation at the optimized impeller (with 48 evaluations) decreased further. Finally, cavitation was nearly eliminated at the end of optimization process (i.e., 60 evaluations).

The optimization benefits in overall efficiency and flow characteristic parameters are demonstrated by the significantly improved flow in the impeller and diffuser tube.

As shown in Figure 12, the optimized impeller geometry had a better matched flow compared with the original, and the impeller's exit separated flow was suppressed. As a result, the local mechanical dissipation decreased and the local entropy production dropped, as seen in Figure 13a. This improved the local static pressure (Figure 13b) and suppressed rises in temperature (Figure 13c), both of which are favorable for impeller cavitation mitigation. Thus, the local vapor fraction was significantly reduced and nearly vanished. As shown in Figure 13d, the scale and intensity of impeller cavitation was almost eliminated, with the maximum vapor volume fraction on the blade suction side reduced from 0.365 to 0.172 .
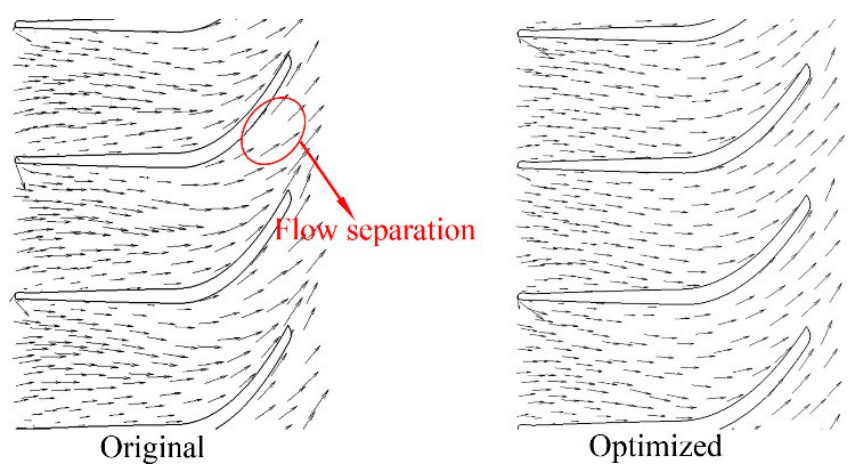

Figure 12. Comparison of impeller flow separation at a $90 \%$ span. 

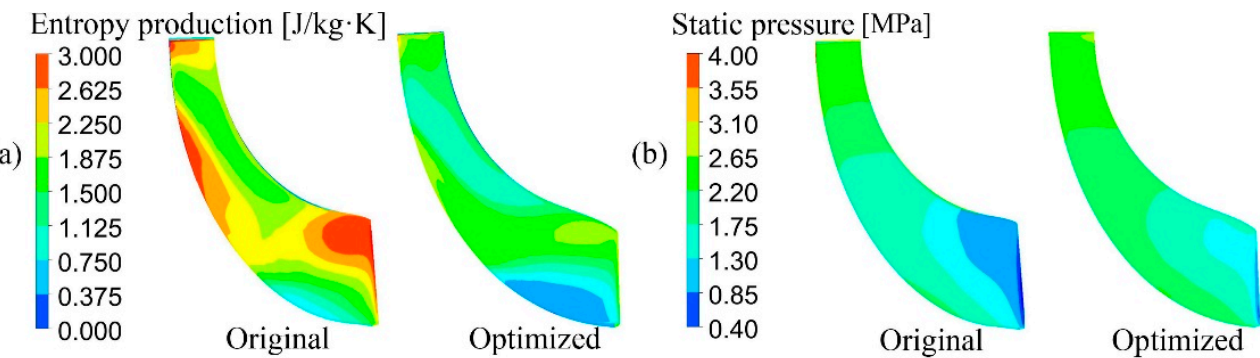

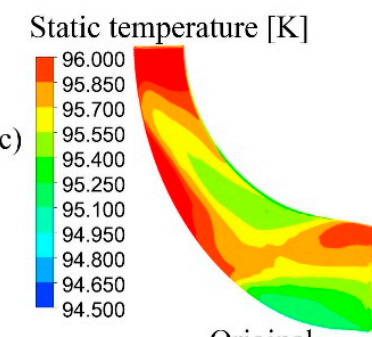

Original

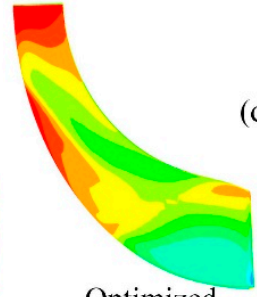

Optimized
Vapor volume fraction

(d)
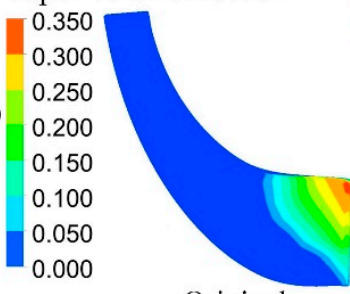

Original

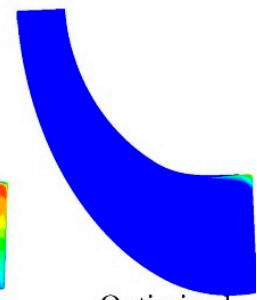

Optimized

Figure 13. Comparison of impeller entropy production, static pressure, temperature and vapor fraction: (a) entropy production; (b) static pressure; (c) static temperature; (d) vapor volume fraction.

In comparison with the original diffuser tube, the optimized geometry largely reduced the diffuser tube circumferential velocity component (Figure 14a), and the optimized streamlines were much less rope-like, especially around the centerline (Figure 14b). Consequently, the local mechanical dissipation decreased in terms of entropy production (Figure 15a), and the local pressure drops and temperature rises reduced as well (Figure 15b,c), which is favorable for diffuser tube cavitation mitigation as it largely reduces the local vapor fraction to a minimum. As a result, the intensive vapor fraction around the centerline was largely reduced. As is shown in Figure 15d, the maximal vapor volume fraction in the diffuser tube reduced from 0.387 to 0.121 .

(a)

Circumferential velocity $[\mathrm{m} / \mathrm{s}]$
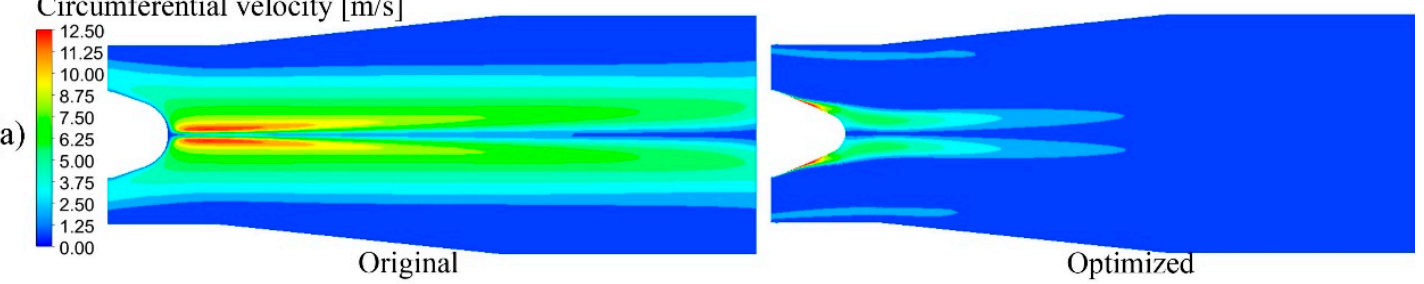

(b)

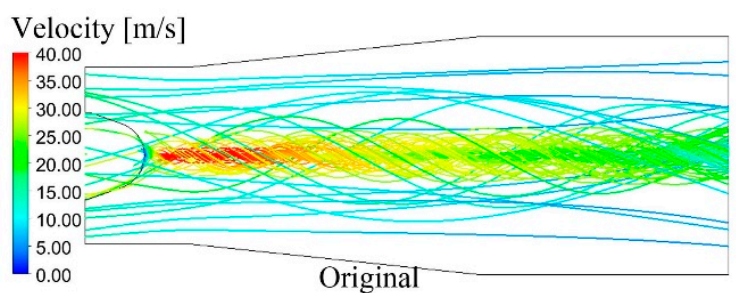

Original

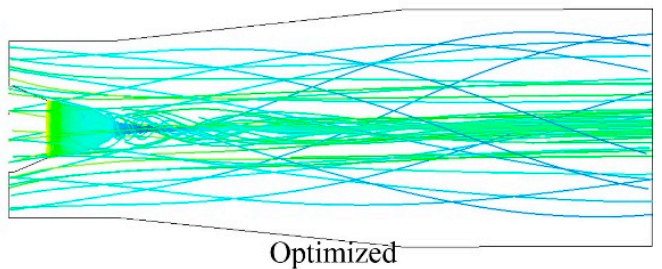

Figure 14. Comparison of diffuser tube swirling flow: (a) circumferential velocity contour; (b) streamline/velocity contour. 

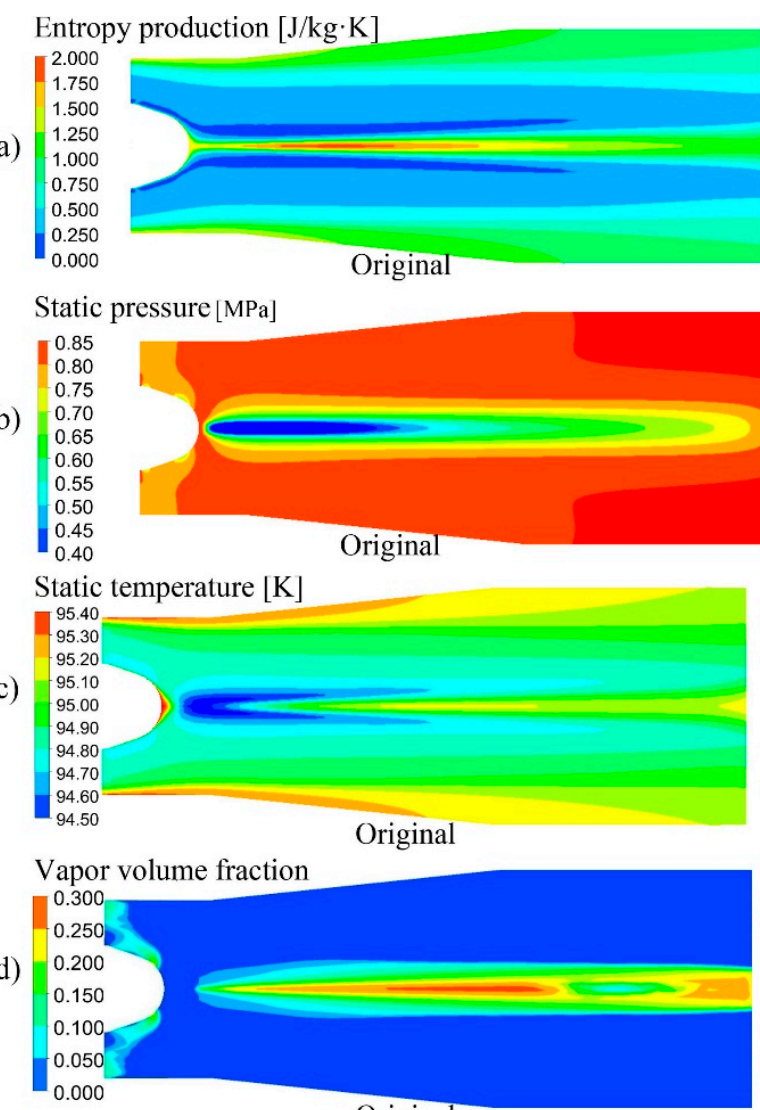

Original
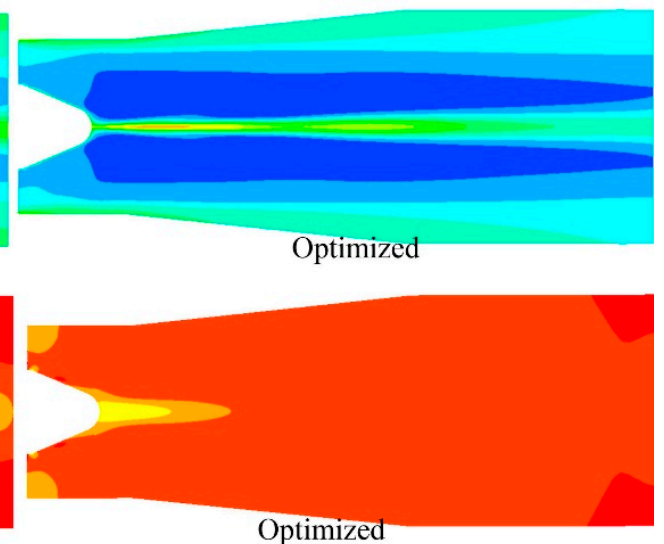

Optimized

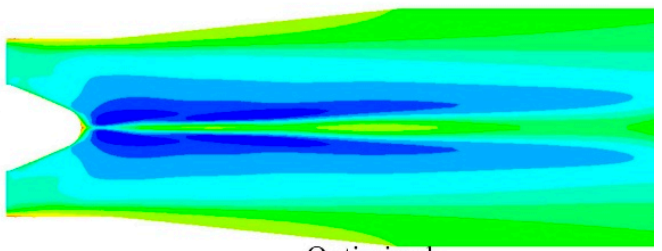

Optimized

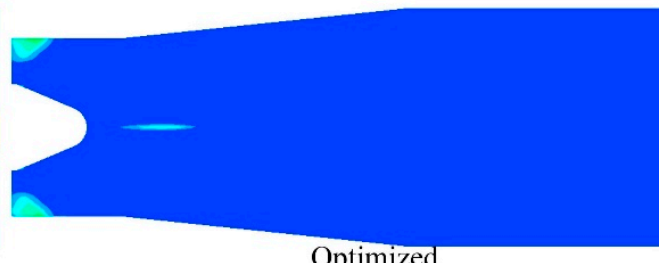

Optimized

Figure 15. Comparison of diffuser entropy production, static pressure, temperature and vapor fraction: (a) entropy production; (b) static pressure; (c) static temperature; (d) volume vapor fraction.

\section{Concluding Remarks}

In the present work, efforts have been made towards cavitation suppression in a liquid turbine expander through collaborative optimization of an impeller and its fairing cone shapes. The following conclusions can be made:

(1) The cavitating flow in the originally designed turbine expander was explored. The impeller cavitation was located around the trailing edge of the impeller blade. The reason for this is that the impeller blade trailing-edge geometry did not match the impeller exiting flow, which induced local flow separation. Such flow separation causes local mechanical head dissipation and, subsequently, local static pressure drops and temperature rises. The combined effect of low static pressure and a high static temperature is responsible for the local cavitation. In the diffuser tube, cavitation was located in two regions: the smaller-scale cavitation formed at the impeller blade trailing, and the larger-scale intensive cavitation formed around the centerline of the diffuser tube. The former resulted from the propagation of the impeller cavitation, while the latter arose from the swirling vortex flow in the diffuser tube. Such swirling flow causes considerable flow loss, subsequently causing local pressure drops and temperature rises, both of which were responsible for the swirling-flow-associated cavitation around the diffuser tube center core region.

(2) To effectively suppress cavitation in liquid turbine expanders, a well-suited CFD-based design optimization method was developed. The optimization variables were identified and a flexible variation of the impeller and fairing cone geometries was simultaneously realized where the former was tuned on both levels of the 2D meridian profile and the 3D blade geometry, and the latter was tuned in 2D dimensions. A novel objective function was deliberately established by allowing for both swirling flow and cavitation characteristics, driving the optimizer to 
search for deswirling and cavitation-resistant geometries. A kriging surrogate model with an adaptive sampling strategy and the CCEA were incorporated to solve the highly nonlinear design optimization globally with a high convergence rate.

(3) The optimized impeller meridian profile and blade shape and fairing cone geometry all deviated significantly from the original, and were proven favorable for combing the impeller and diffuser tube flow, thus mitigating the cavitation: the impeller trailing edge separated flow was effectively suppressed, the local vapor fraction vanished, the diffuser tube high-swirl flow was significantly deswirled and the intensive vapor fraction around the centerline was largely reduced.

Optimized impeller geometry permits better matched flow, where the local separated flow separation around an impeller exit is effectively suppressed and local mechanical dissipation is subsequently decreased in terms of entropy production. The reduced local pressure drops and temperature rises are favorable for impeller cavitation mitigation, causing the vapor fraction around the impeller exit to vanish.

In comparison with the original diffuser tube, the optimized geometry largely reduces the diffuser tube circumferential velocity component, and the original rope-like swirling flow is effectively deswirled. Consequently, local mechanical dissipation decreases in terms of entropy production as the local static pressure rises and the local static temperature drops. These conditions are favorable for diffuser tube cavitation mitigation and reduction of the local vapor fraction to a minimum.

Author Contributions: P.S., J.S. conceived and performed the study, P.S. wrote the paper, J.S. reviewed and edited the manuscript. All authors have read and agreed to the published version of the manuscript.

Funding: The work is supported by the National Natural Science Foundation of China under Grant (Project No. 51576148 and 51606141) and China Postdoctoral Science Foundation under Grant 2016M602817, which are highly acknowledged by the authors.

Conflicts of Interest: The authors declare no conflict of interest.

\section{Nomenclature}

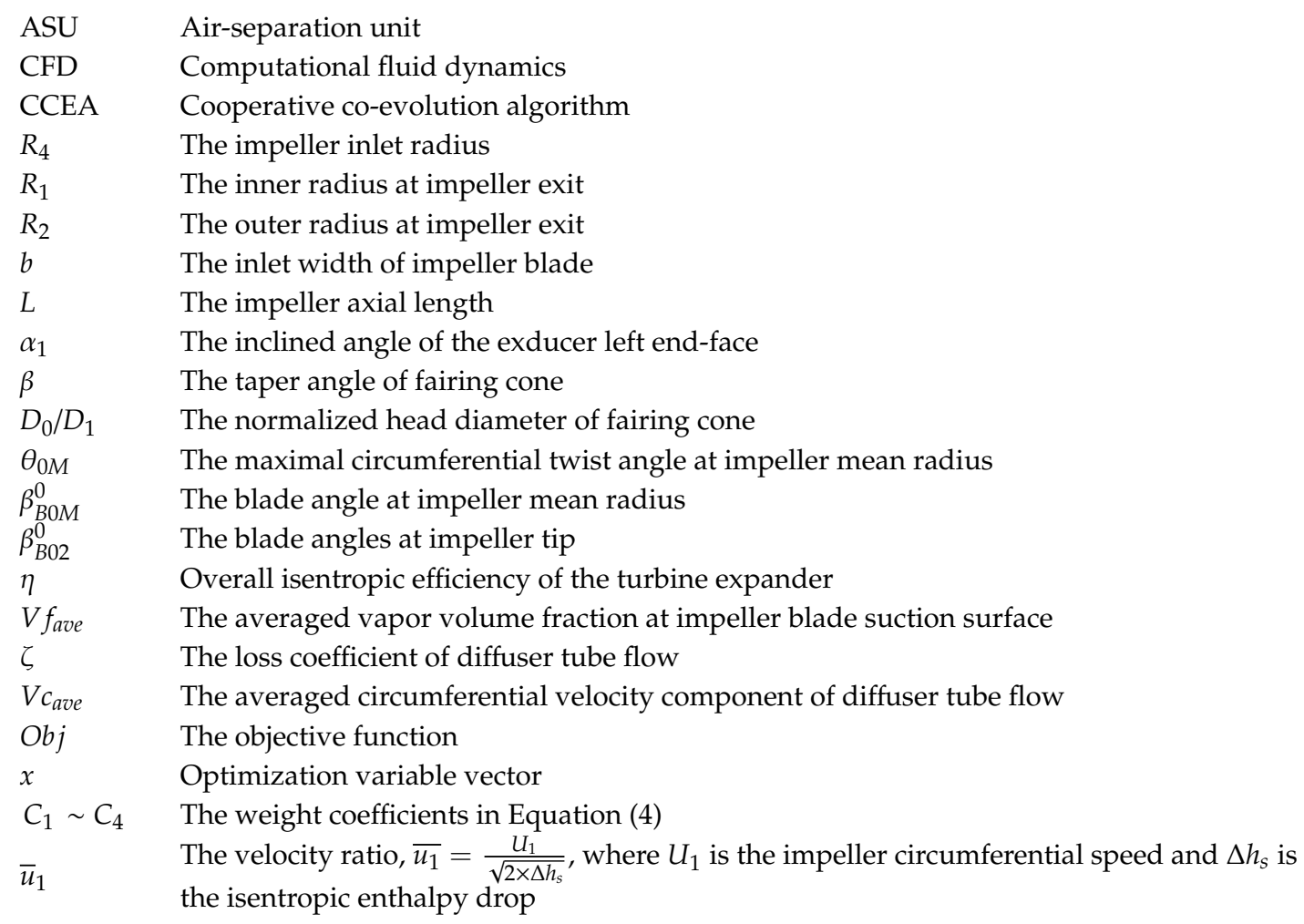




\section{References}

1. Chiu, C.H.; Kimmel, H.E. Turbo-expander technology development for LNG plants. In Proceedings of the 13th International Conference \& Exhibition on Liquefied Natural Gas, Seoul, Korea, 13-16 May 2001.

2. Kimmel, H. Speed controlled turbines for power recovery in cryogenic and chemical processing. World Pumps 1997, 1997, 46-49. [CrossRef]

3. Hudson, H.M.; Wilkinson, J.; Cuellar, K.; Pierce, M.C. Integrated liquids recovery technology improves LNG production efficiency. In Proceedings of the 82nd Annual Convention of the Gas Processors Association, San Antonio, TX, USA, 11 March 2003.

4. Johnson, L.L. Improvement of natural gas liquefaction processes by using liquid turbines. Fuel Energy Abstr. 1998, 39, 51.

5. Haesloop, B.; Kimmel, H. Improved Cryogenic Gas Processing Efficiency Due to Advancements in Liquid Turbine Expanders; American Institute of Chemical Engineers: New York, NY, USA, 1998.

6. Habets, G.; Kimmel, H. Economics of cryogenic turbine expanders. Gas 1998, 1, 4.

7. Hosangadi, A.; Ahuja, V.; Ungewitter, R.J.; Busby, J. Numerical study of a flat plate inducer: Comparison of performance in liquid hydrogen and water. In Proceedings of the 42nd AIAA/ASME/SAE/ASEE Joint Propulsion Conference \& Exhibit, Sacramento, CA, USA, 9-12 July 2006. Paper No. AIAA 2006-5070.

8. Hosangadi, A.; Ahuja, V.; Ungewitter, R.J. Simulations of a liquid hydrogen inducer at low-flow off-design flow conditions. In Proceedings of the 41st AIAA/ASME/SAE/ASEE Joint Propulsion Conference \& Exhibit, Tucson, AZ, USA, 10-13 July 2005. Paper No. AIAA-2005-4450.

9. Goncalvès, E.; Patella, R.F.; Rolland, J.; Pouffary, B.; Challier, G. Thermodynamic effect on a cavitating inducer in liquid hydrogen. J. Fluids Eng. 2010, 132, 111305. [CrossRef]

10. Kumar, P.; Saini, R. Study of cavitation in hydro turbines-A review. Renew. Sustain. Energy Rev. 2010, 14, 374-383. [CrossRef]

11. Singhal, A.K.; Athavale, M.M.; Li, H.; Jiang, Y. Mathematical basis and validation of the full cavitation model. J. Fluids Eng. 2002, 124, 617-624. [CrossRef]

12. Kunz, R.F.; Boger, D.A.; Stinebring, D.R.; Chyczewski, T.S.; Lindau, J.W.; Gibeling, H.J.; Venkateswaran, S.; Govindan, T.R. A preconditioned Navier-Stokes method for two-phase flows with application to cavitation prediction. Comput. Fluids 2000, 29, 849-875. [CrossRef]

13. Medvitz, R.B.; Kunz, R.F.; Boger, D.A.; Lindau, J.W.; Yocum, A.M.; Pauley, L.L. Performance analysis of cavitating flow in centrifugal pumps using multiphase CFD. J. Fluids Eng. 2002, 124, 377-383. [CrossRef]

14. Gerber, A. A CFD model for devices operating under extensive cavitation conditions. In Proceedings of the ASME 2002 International Mechanical Engineering Congress and Exposition, New Orleans, LA, USA, 17-22 November 2002; pp. 341-349, Paper No. IMECE2002-39315.

15. Bakir, F.; Rey, R.; Gerber, A.G.; Belamri, T.; Hutchinson, B. Numerical and experimental investigations of the cavitating behavior of an inducer. Int. J. Rotating Mach. 2004, 10, 15-25. [CrossRef]

16. Brennen, C.E. Hydrodynamics of Pumps; Oxford Science Publications: London, UK, 1994; p. 81.

17. Iliescu, M.S.; Ciocan, G.D.; Avellan, F. 3D PIV and LDV measurements at the outlet of a Francis turbine draft tube. In Proceedings of the ASME 2002 Joint US-European Fluids Engineering Division Conference, Montreal, QC, Canada, 14-18 July 2002; pp. 311-316.

18. Ciocan, G.D.; Iliescu, M.S.; Vu, T.C.; Nennemann, B.; Avellan, F. Experimental study and numerical simulation of the FLINDT draft tube rotating vortex. J. Fluids Eng. 2007, 129, 146-158. [CrossRef]

19. Ruprecht, A.; Helmrich, T.; Aschenbrenner, T.; Scherer, T. Simulation of vortex rope in a turbine draft tube. In Proceedings of the 22nd IAHR Symposium on Hydraulic Machinery and Systems, Stockholm, Sweden, 29 June-2 July 2004; pp. 9-12.

20. Paik, J.; Sotiropoulos, F.; Sale, M.J. Numerical simulation of swirling flow in complex hydroturbine draft tube using unsteady statistical turbulence models. J. Hydraul. Eng. 2005, 131, 441-456. [CrossRef]

21. Susan-Resiga, R.; Muntean, S.; Stein, P.; Avellan, F. Axisymmetric swirling flow simulation of the draft tube vortex in Francis turbines at partial discharge. Int. J. Fluid Mach. Syst. 2009, 2, 295-302. [CrossRef]

22. Susan-Resiga, R.; Ciocan, G.D.; Anton, I.; Avellan, F. Analysis of the swirling flow downstream a Francis turbine runner. J. Fluids Eng. 2006, 128, 177-189. [CrossRef] 
23. Tridon, S.; Barre, S.; Ciocan, G.D.; Tomas, L. Experimental analysis of the swirling flow in a Francis turbine draft tube: Focus on radial velocity component determination. Eur. J. Mech. B/Fluids 2010, 29, 321-335. [CrossRef]

24. Iliescu, M.S.; Ciocan, G.D.; Avellan, F. Analysis of the cavitating draft tube vortex in a Francis turbine using particle image velocimetry measurements in two-phase flow. J. Fluids Eng. 2008, 130, 021105. [CrossRef]

25. Escaler, X.; Egusquiza, E.; Farhat, M.; Avellan, F.; Coussirat, M. Detection of cavitation in hydraulic turbines. Mech. Syst. Signal Process. 2006, 20, 983-1007. [CrossRef]

26. Zhang, H.; Zhang, L. Numerical simulation of cavitating turbulent flow in a high head Francis turbine at part load operation with Open FOAM. Pocedia Eng. 2012, 31, 156-165. [CrossRef]

27. Susan-Resiga, R.; Vu, T.C.; Muntean, S.; Ciocan, G.D.; Nennemann, B. Jet control of the draft tube vortex rope in Francis turbines at partial discharge. In Proceedings of the 23rd IAHR Symposium on Hydraulic Machinery and Systems, Yokohama, Japan, 24-28 October 2006; pp. 17-21.

28. Hočevar, M.; Širok, B.; Blagojevič, B. Prediction of cavitation vortex dynamics in the draft tube of a francis turbine using radial basis neural networks. Neural Comput. Appl. 2005, 14, 229-234. [CrossRef]

29. Galván, S.; Reggio, M.; Guibault, F. Inlet Velocity Profile Optimization of the Turbine 99 Draft Tube. In Proceedings of the ASME 2013 Fluids Engineering Division Summer Meeting, Incline Village, NV, USA, 7-11 July 2013; pp. V01AT2A007-V01AT02A.

30. Ruprecht, A.; Eisinger, R.; Göde, E. Innovative design environments for hydro turbine components. Bern HYDRO 2000.

31. Eisinger, R.; Ruprecht, A. Automatic shape optimization of hydro turbine components based on CFD. Task Q. 2001, 6, 101-111.

32. Marjavaara, B.D.; Lundström, T.S. Redesign of a sharp heel draft tube by a validated CFD-optimization. Int. J. Numer. Methods Fluids. 2006, 50, 911-924. [CrossRef]

33. Sergio, G.; Carlos, R.; Pacheco, J.; Mendoza, C.; Toledo, M. Optimization methodology assessment for the inlet velocity profile of a hydraulic turbine draft tube: Part II-performance evaluation of draft tube model. J. Glob. Optim. 2013, 55, 729-749. [CrossRef]

34. Cervone, A.; Pace, G.; Torre, L.; Pasini, A.; Bartolini, S.; Agnesi, L.; d'Agostino, L. Effects of the leading edge shape on the performance of an axial three bladed inducer. In Proceedings of the 14th International Symposium on Transport Phenomena and Dynamics of Rotating Machinery, Honolulu, HI, USA, 27 February-2 March 2012.

35. Coutier-Delgosha, O.; Reboud, J.; Fortes-Patella, R. Numerical study of the effect of the leading edge shape on cavitation around inducer blade sections. JSME Int. J. Ser. B Fluids Therm. Eng. 2002, 45, 678-685. [CrossRef]

36. Pierrat, D.; Gros, L.; Pintrand, G.; Le Fur, B.; Gyomlai, P. Experimental and numerical investigations of leading edge cavitation in a helico-centrifugal pump. In Proceedings of the 12th International Symposium of Transport Phenomena and Dynamics on Rotating Machinery, Honolulu, HI, USA, 17-22 February 2008.

37. Kamijo, K.; Yoshida, M.; Tsujimoto, Y. Hydraulic and mechanical performance of LE-7 LOX pump inducer. J. Propuls. Power 1993, 9, 819-826. [CrossRef]

38. Hong, S.S.; Kim, D.J.; Kim, J.S.; Choi, C.H.; Kim, J. Study on inducer and impeller of a centrifugal pump for a rocket engine turbopump. Proceedings of the Institution of Mechanical Engineers. Part C J. Mech. Eng. Sci. 2013, 227, 311-319. [CrossRef]

39. Jakobsen, J.K.; Keller, R., Jr. Liquid Rocket Engine Turbopump Inducers; NASA Report: SP-8052; NASA: Washington, DC, USA, 1971.

40. Utturkar, Y.; Wu, J.; Wang, G.; Shyy, W. Recent progress in modeling of cryogenic cavitation for liquid rocket propulsion. Prog. Aerosp. Sci. 2005, 41, 558-608. [CrossRef]

41. Hosangadi, A.; Ahuja, V.; Ungewitter, R.J.; Busby, J. Analysis of thermal effects in cavitating liquid hydrogen inducers. J. Propuls. Power 2007, 23, 1225-1234. [CrossRef]

42. He, Z.L.; Ge, Y.Z.; Sun, J.J.; Wang, K. Numerical Study of Cavitating Behavior in a Cryogenic Liquid Turbine. In Proceedings of the ASME Turbo Expo 2012: Turbomachinery Technical Conference and Exposition, Copenhagen, Denmark, 11-15 June 2012. ASME Paper GT2012-68774.

43. Li, K.Q.; Sun, J.J.; Fu, J.T.; Song, P. Design and Numerical Flow Analysis of a LNG Power Recovery Turbine. In Proceedings of the ASME Turbo Expo 2013: Turbomachinery Technical Conference and Exposition, San Antonio, TX, USA, 3-7 June 2013. ASME Paper GT2013-94900. 
44. Song, P.; Sun, J.J.; Wang, K. Swirling and cavitating flow suppression in a cryogenic liquid turbine expander through geometric optimization. Proc. Inst. Mech. Eng. Part A J. Power Energy 2015, 229, 628-646. [CrossRef]

45. Song, P.; Sun, J.J.; Li, K.Q.; Wang, K.; Huo, C.J. Numerical Study of Cavitating Flow in Two-Phase LNG Expander. In Proceedings of the ASME Turbo Expo 2016: Turbomachinery Technical Conference and Expositio, Seoul, Korea, 13-17 June 2016. ASME Paper GT2016-56780.

46. Wang, K.; Sun, J.J.; Song, P.; Huo, C.J. Influence of Impeller Fairing Cone Geometry on Cavitating Flow Behavior in a Cryogenic Liquid Turbine Expander. In Proceedings of the ASME Turbo Expo 2016: Turbomachinery Technical Conference and Expositio, Seoul, Korea, 13-17 June 2016. ASME Paper GT2016-56778.

47. Tao, R.; Xiao, R.F.; Yang, W.; Wang, F.J.; Liu, W.C. Optimization for cavitation inception performance of pump-turbine in pump mode based on genetic algorithm. Math. Probl. Eng. 2014, 2014, 1-9. [CrossRef]

48. CFX 12.1 Help Manual; Ansys Inc.: Canonsburg, PA, USA.

49. Goel, T.; Thakur, S.; Haftka, R.T.; Shyy, W.; Zhao, J. Surrogate model-based strategy for cryogenic cavitation model validation and sensitivity evaluation. Int. J Numer. Methods Fluids 2008, 58, 969-1007. [CrossRef]

50. Chen, Y.; Heister, S.D. A numerical treatment for attached cavitation. J. Fluids Eng. 1994, 116, $613-618$. [CrossRef]

51. Zwart, P.J.; Gerber, A.G.; Belamri, T. A two-phase model for predicting cavitation dynamics. In Proceedings of the International Conference on Multiphase Flow (ICMF 2004), Yokohama, Japan, 30 May-3 June 2004; Volume 152.

52. Hosangadi, A.; Ahuja, V.; Ungewitter, R.J. Simulations of cavitating flows in turbopumps. J. Propuls. Power 2004, 20, 604-611. [CrossRef]

53. Hord, J. Cavitation in Liquid Cryogens, II-Hydrofoil; NASA CR-2156; NASA: Washington, DC, USA, 1973.

54. Galván, S.; Guibault, F.; Reggio, M. Numerical validation of different CFD k-e turbulent models using FLUENT code. In Proceedings of the Third IAHR/ERCOFTAC Workshop on Draft Tube Flow, Porjus, Sweden, 8-9 December 2005. Paper No. 4.

55. Galván, S.; Reggio, M.; Guibault, F. Assessment study of k- $\varepsilon$ turbulence models and near-wall modeling for steady state swirling flow analysis in draft tube using FLUENT. Eng. Appl. Comput. Fluid Mech. 2011, 5, 459-478. [CrossRef]

56. Wang, K.; Sun, J.; Song, P. Experimental study of cryogenic liquid turbine expander with close-loop liquefied nitrogen system. Cryogenics 2015, 67, 4-14. [CrossRef]

57. Balje, O. Turbomachines: A Guide to Design Selection and Theory; John Willey \& Son Inc.: Hoboken, NJ, USA, 1981.

58. Song, P.; Sun, J.J.; Wang, K. Axial flow compressor blade optimization through flexible shape tuning by means of cooperative co-evolution algorithm and adaptive surrogate model. Proc. Inst. Mech. Eng. Part A J. Power Energy 2014, 228, 782-798. [CrossRef]

(C) 2019 by the authors. Licensee MDPI, Basel, Switzerland. This article is an open access article distributed under the terms and conditions of the Creative Commons Attribution (CC BY) license (http://creativecommons.org/licenses/by/4.0/). 